\title{
Nuclear phase transition and thermodynamic instabilities in dense nuclear matter
}

\author{
A. Lavagno ${ }^{a}$ \\ ${ }^{1}$ Department of Applied Science and Technology, Politecnico di Torino, I-10129 Torino, Italy \\ ${ }^{2}$ Istituto Nazionale di Fisica Nucleare (INFN), Sezione di Torino, I-10126 Torino, Italy
}

\begin{abstract}
We study the presence of thermodynamic instabilities in a nuclear medium at finite temperature and density where nuclear phase transitions can take place. Such a phase transition is characterized by pure hadronic matter with both mechanical instability (fluctuations on the baryon density) that by chemical-diffusive instability (fluctuations on the electric charge concentration). Similarly to the liquid-gas phase transition, the nucleonic and the $\Delta$-matter phase have a different isospin density in the mixed phase. In the liquid-gas phase transition, the process of producing a larger neutron excess in the gas phase is referred to as isospin fractionation. A similar effects can occur in the nucleon- $\Delta$ matter phase transition due essentially to a $\Delta^{-}$excess in the $\Delta$-matter phase in asymmetric nuclear matter. In this context we also discuss the relevance of $\Delta$-isobar and hyperon degrees of freedom in the bulk properties of the protoneutron stars at fixed entropy per baryon, in the presence and in the absence of trapped neutrinos.
\end{abstract}

\section{Introduction}

One of the very interesting aspects in nuclear astrophysics and in the heavy-ion collisions experiments is a detailed study of the thermodynamical properties of strongly interacting nuclear matter away from the nuclear ground state. In this direction, many efforts have been focused on searching for possible phase transitions at finite temperature in dense nuclear matter. The knowledge of the nuclear equation of state (EOS) of dense matter at finite temperature plays a crucial role in the determination of the structure and in the evolution of the protoneutron star (PNS) [1-3]. A PNS is formed in a stellar remnant after a successful core-collapse supernova explosion of a star with a mass smaller than about 20 solar masses and in the first seconds of its evolution it is a very hot (temperature of up to $50 \mathrm{MeV}$ ), lepton rich and $\beta$-stable object and a lepton concentration typical of the pre-supernova matter [1,2].

In this paper, we are going to study a EOS at finite temperature and density by means of a relativistic mean-field model with the inclusion $\Delta(1232)$-isobars [4-6] and by requiring the Gibbs conditions on the global conservation of baryon number and net electric charge. In regime of finite values of density and temperature, a state of high density resonance matter may be formed and the $\Delta(1232)$ isobar degrees of freedom are expected to play a central role in relativistic heavy ion collisions and in the physics of compact stars [7,8]. Transport model calculations and experimental results indicate that an excited state of baryonic matter is dominated by the $\Delta$-resonance at the energy from the BNL

ae-mail: andrea.lavagno@polito.it 
Alternating Gradient Synchrotron (AGS) to RHIC [9, 10]. Moreover, in the framework of the nonlinear Walecka model, it has been predicted that a phase transition from nucleonic matter to $\Delta$-excited nuclear matter can take place and the occurrence of this transition sensibly depends on the $\Delta$-meson coupling constants $[11,12]$.

\section{Relativistic nuclear equation of state and stability conditions}

In this section, we start by introducing the hadronic equation of state (EOS) in the framework of a relativistic mean-field theory. In this investigation we include all the baryon octet in order to reproduce the chemical composition of the PNS at high baryon chemical potential. We also take into account of leptons particle by fixing the lepton fraction $Y_{L}=Y_{e}+Y_{v_{e}}=\left(\rho_{e}+\rho_{v_{e}}\right) / \rho_{B}$, where $\rho_{e}, \rho_{v_{e}}$ and $\rho_{B}$ are the electron, neutrino and baryon number densities, respectively. This is because, in the first stage of PNS evolution, electrons and neutrinos are trapped inside the stellar matter and, therefore, the lepton number must be conserved until neutrinos escape out of the PNS [2].

The Lagrangian density can be written in term of the hadronic $[4,13,14]$ plus leptonic component, as follow:

$$
\begin{aligned}
& \mathcal{L}_{\text {tot }}=\mathcal{L}_{\text {octet }}+\mathcal{L}_{\Delta}+\mathcal{L}_{l}=\sum_{k} \bar{\psi}_{k}\left[i \gamma_{\mu} \partial^{\mu}-\left(M_{k}-g_{\sigma k} \sigma\right)-g_{\omega k} \gamma_{\mu} \omega^{\mu}-g_{\rho k} \gamma^{\mu} \vec{\tau} \cdot \vec{\rho}_{\mu}\right] \psi_{k} \\
& +\frac{1}{2}\left(\partial_{\mu} \sigma \partial^{\mu} \sigma-m_{\sigma}^{2} \sigma^{2}\right)-U(\sigma)+\frac{1}{2} m_{\omega}^{2} \omega_{\mu} \omega^{\mu}+\frac{1}{2} m_{\rho}^{2} \vec{\rho}_{\mu} \cdot \vec{\rho}^{\mu}-\frac{1}{4} F_{\mu \nu} F^{\mu v}-\frac{1}{4} \vec{G}_{\mu \nu} \vec{G}^{\mu v} \\
& +\bar{\psi}_{\Delta v}\left[i \gamma_{\mu} \partial^{\mu}-\left(M_{\Delta}-g_{\sigma \Delta} \sigma\right)-g_{\omega \Delta} \gamma_{\mu} \omega^{\mu}\right] \psi_{\Delta}^{v}+\sum_{l} \bar{\psi}_{l}\left[i \gamma_{\mu} \partial^{\mu}-m_{l}\right] \psi_{l},
\end{aligned}
$$

where the sums over $k$ and $l$ are over the baryon octet and lepton particles, respectively; $\psi_{\Delta}^{v}$ is the Rarita-Schwinger spinor for the $\Delta$-isobars $\left(\Delta^{++}, \Delta^{+}, \Delta^{0}, \Delta^{-}\right)$. The field strength tensors for the vector mesons are given by the usual expressions $F_{\mu \nu} \equiv \partial_{\mu} \omega_{v}-\partial_{\nu} \omega_{\mu}, \vec{G}_{\mu \nu} \equiv \partial_{\mu} \vec{\rho}_{\nu}-\partial_{\nu} \vec{\rho}_{\mu}$, and $U(\sigma)$ is a nonlinear potential of $\sigma$ meson

$$
U(\sigma)=\frac{1}{3} a \sigma^{3}+\frac{1}{4} b \sigma^{4}
$$

usually introduced to achieve a reasonable compression modulus for equilibrium nuclear matter.

The field equations in a mean field approximation are

$$
\begin{aligned}
& {\left[i \gamma_{\mu} \partial^{\mu}-\left(M_{k}-g_{\sigma k} \sigma\right)-g_{\omega k} \gamma^{0} \omega-g_{\rho k} \gamma^{0} \tau_{3} \rho\right] \psi_{k}=0,} \\
& {\left[i \gamma_{\mu} \partial^{\mu}-\left(M_{\Delta}-g_{\sigma \Delta} \sigma\right)-g_{\omega \Delta} \gamma^{0} \omega\right] \psi_{\Delta}^{v}=0,} \\
& m_{\sigma}^{2} \sigma+a \sigma^{2}+b \sigma^{3}=\sum_{k} g_{\sigma k} \rho_{k}^{S}, \\
& m_{\omega}^{2} \omega=\sum_{k} g_{\omega k} \rho_{k}^{B}, \\
& m_{\rho}^{2} \rho=g \sum_{k} g_{\rho k} t_{3 k} \rho_{k}^{B},
\end{aligned}
$$

where $\sigma=\langle\sigma\rangle, \omega=\left\langle\omega^{0}\right\rangle$ and $\rho=\left\langle\rho_{3}^{0}\right\rangle$ are the nonvanishing expectation values of mesons fields. The $\rho_{k}^{B}$ and $\rho_{k}^{S}$ are the baryon density and the baryon scalar density, respectively. They are given by

$$
\begin{aligned}
& \rho_{i}^{B}=\gamma_{i} \int \frac{\mathrm{d}^{3} k}{(2 \pi)^{3}}\left[n_{i}(k)-\bar{n}_{i}(k)\right], \\
& \rho_{i}^{S}=\gamma_{i} \int \frac{\mathrm{d}^{3} k}{(2 \pi)^{3}} \frac{M_{i}^{*}}{E_{i}^{*}}\left[n_{i}(k)+\bar{n}_{i}(k)\right],
\end{aligned}
$$


where $\gamma_{i}$ is the degeneracy spin factor $\left(\gamma_{N}=2\right.$ and $\left.\gamma_{\Delta}=4\right)$ and $n_{i}(k)$ and $\bar{n}_{i}(k)$ are the fermion particle and antiparticle distribution functions.

The nucleon effective energy is defined as $E_{i}{ }^{*}(k)=\sqrt{k^{2}+M_{i}{ }^{* 2}}$, where $M_{i}{ }^{*}=M_{i}-g_{\sigma B} \sigma$. The effective chemical potentials $\mu_{i}^{*}$ are given in terms of the meson fields as follows

$$
\mu_{i}^{*}=\mu_{i}-g_{\omega B} \omega-\tau_{3 i B} g_{\rho B} \rho,
$$

where $\mu_{i}$ are the thermodynamical chemical potentials $\left(\mu_{i}=\partial \epsilon / \partial \rho_{i}\right)$.

The further conditions, required for the $\beta$-stable chemical equilibrium and charge neutrality, can be written as

$$
\begin{aligned}
& \mu_{\Lambda}=\mu_{\Sigma^{0}}=\mu_{\Xi^{0}}=\mu_{\Delta^{0}}=\mu_{n}, \\
& \mu_{\Sigma^{-}}=\mu_{\Xi^{-}}=\mu_{\Delta^{-}}=\mu_{n}+\mu_{e}, \\
& \mu_{p}=\mu_{\Sigma^{+}}=\mu_{\Delta^{+}}=\mu_{n}-\mu_{e}, \\
& \mu_{\Delta^{++}}=\mu_{n}-2 \mu_{e} ; \\
& \rho_{p}+\rho_{\Sigma^{+}}-\rho_{\Sigma^{-}}-\rho_{\Xi^{-}}+\rho_{\Delta^{+}}-\rho_{\Delta^{-}}+2 \rho_{\Delta^{++}}-\rho_{e}=0 .
\end{aligned}
$$

In the case of trapped neutrinos, the new equalities are obtained by the replacement of $\mu_{e} \rightarrow \mu_{e}-\mu_{v_{e}}$. The total entropy per baryon is calculated using $s=\left(S_{B}+S_{l}\right) /\left(T \rho_{B}\right)$, where $S_{B}=P_{B}+\epsilon_{B}-\sum_{i=B} \mu_{i} \rho_{i}$ and $S_{l}=P_{l}+\epsilon_{l}-\sum_{i=l} \mu_{i} \rho_{i}$, and the sums are extended over all the baryons and leptons species.

The thermodynamical quantities can be obtained from the thermodynamic potential in the standard way $[15,16]$. More explicitly, the baryon pressure $P_{B}$ and the energy density $\epsilon_{B}$ can be written as

$$
\begin{aligned}
P_{B}= & \frac{2}{3} \sum_{i} \int \frac{\mathrm{d}^{3} k}{(2 \pi)^{3}} \frac{k^{2}}{E_{i}^{*}(k)}\left[n_{i}(k)+\bar{n}_{i}(k)\right]-\frac{1}{2} m_{\sigma}^{2} \sigma^{2} \\
& -U(\sigma)+\frac{1}{2} m_{\omega}^{2} \omega^{2}+\frac{1}{2} m_{\rho}^{2} \rho^{2}, \\
\epsilon_{B}= & 2 \sum_{i} \int \frac{\mathrm{d}^{3} k}{(2 \pi)^{3}} E_{i}^{*}(k)\left[n_{i}(k)+\bar{n}_{i}(k)\right]+\frac{1}{2} m_{\sigma}^{2} \sigma^{2} \\
& +U(\sigma)+\frac{1}{2} m_{\omega}^{2} \omega^{2}+\frac{1}{2} m_{\rho}^{2} \rho^{2} .
\end{aligned}
$$

Here and in the following, we focus our investigation by considering the so-called GM3 [4] and the SFHo parameter sets $[17,18]$. The implementation of hyperon degrees of freedom comes from determination of the corresponding meson-hyperon coupling constants that have been fitted to hypernuclear properties.

Concerning the stability conditions, we are dealing with the study of a multi-component system at finite temperature and density with two conserved charges: baryon number and electric charge. For such a system, the Helmholtz free energy density $F$ can be written as [19]

$$
F\left(T, \rho_{B}, \rho_{C}\right)=-P\left(T, \mu_{B}, \mu_{C}\right)+\mu_{B} \rho_{B}+\mu_{C} \rho_{C},
$$

with

$$
\mu_{B}=\left(\frac{\partial F}{\partial \rho_{B}}\right)_{T, \rho_{C}}, \quad \mu_{C}=\left(\frac{\partial F}{\partial \rho_{C}}\right)_{T, \rho_{B}} .
$$

In a system with $N$ different particles, the particle chemical potentials are expressed as the linear combination of the two independent chemical potentials $\mu_{B}$ and $\mu_{C}$ and, as a consequence, $\sum_{i=1}^{N} \mu_{i} \rho_{i}=$ $\mu_{B} \rho_{B}+\mu_{C} \rho_{C}$. 
Assuming the presence of two phases (denoted as $I$ and $I I$, respectively), the system is stable against the separation in two phases if the free energy of a single phase is lower than the free energy in all two phases configuration. The phase coexistence is given by the Gibbs conditions

$$
\begin{aligned}
& \mu_{B}^{I}=\mu_{B}^{I I}, \quad \mu_{C}^{I}=\mu_{C}^{I I}, \\
& P^{I}\left(T, \mu_{B}, \mu_{C}\right)=P^{I I}\left(T, \mu_{B}, \mu_{C}\right) .
\end{aligned}
$$

Therefore, at a given baryon density $\rho_{B}$ and at a given net electric charge density $\rho_{C}=y \rho_{B}$ (with $y=Z / A$ ), the chemical potentials $\mu_{B}$ are $\mu_{C}$ are univocally determined. An important feature of this conditions is that, unlike the case of a single conserved charge, the pressure in the mixed phase is not constant and, although the total $\rho_{B}$ and $\rho_{C}$ are fixed, baryon and charge densities can be different in the two phases. For such a system in thermal equilibrium, the possible phase transition can be characterized by mechanical (fluctuations in the baryon density) and chemical instabilities (fluctuations in the electric charge density) $[11,19]$. As usual the condition of the mechanical stability implies

$$
\rho_{B}\left(\frac{\partial P}{\partial \rho_{B}}\right)_{T, \rho_{C}}>0 .
$$

By introducing the notation $\mu_{i, j}=\left(\partial \mu_{i} / \partial \rho_{j}\right)_{T, P}$ (with $i, j=B, C$ ), the chemical stability for a process at constant $P$ and $T$ can be expressed with the following conditions [11]

$$
\begin{aligned}
& \rho_{B} \mu_{B, B}+\rho_{C} \mu_{C, B}=0, \\
& \rho_{B} \mu_{B, C}+\rho_{C} \mu_{C, C}=0 .
\end{aligned}
$$

Whenever the above stability conditions are not respected, the system becomes unstable and the phase transition takes place $[20,21]$. The coexistence line of a system with one conserved charge becomes in this case a two dimensional surface in $(T, P, y)$ space, enclosing the region where mechanical and diffusive instabilities occur. In analogy with the liquid-gas case, we are going to investigate the existence of a possible phase transition in the nuclear medium by studying the presence of instabilities (mechanical and/or chemical) in the system. The chemical stability condition is satisfied if [11]

$$
\left(\frac{\partial \mu_{C}}{\partial y}\right)_{T, P}>0 \text { or } \begin{cases}\left(\frac{\partial \mu_{B}}{\partial y}\right)_{T, P}<0, & \text { if } y>0, \\ \left(\frac{\partial \mu_{B}}{\partial y}\right)_{T, P}>0, & \text { if } y<0 .\end{cases}
$$

From the analysis of the above chemical potential isobars, we are able to construct the binodal surface relative to the nucleon- $\Delta$ matter phase transition. In Fig. 1, we show the binodal section at $T=50 \mathrm{MeV}$ and $x_{\sigma \Delta}=1.3$ for the GM3 EOS.

The right branch (at lower density) corresponds to the initial phase (I), where the dominant component of the system is given by nucleons. The left branch (II) is related to the final phase at higher densities, where the system is composed primarily by $\Delta$-isobar degrees of freedom ( $\Delta$-dominant phase). In presence of $\Delta$-isobars the phase coexistence region results very different from what obtained in the liquid-gas case, in particular it extends up to regions of negative electric charge fraction and the mixed phase region ends in a point of maximum asymmetry with $y=-1$ (corresponding to a system with almost all $\Delta^{-}$-particles, being antiparticles and pions contribution almost negligible in this regime).

We analyze the phase evolution of the system during the isothermal compression from an arbitrary initial point $A$, indicated in Fig. 1. In this point the system becomes unstable and starts to be energetically favorable the separation into two phases, therefore an infinitesimal $\Delta$-dominant phase appears 


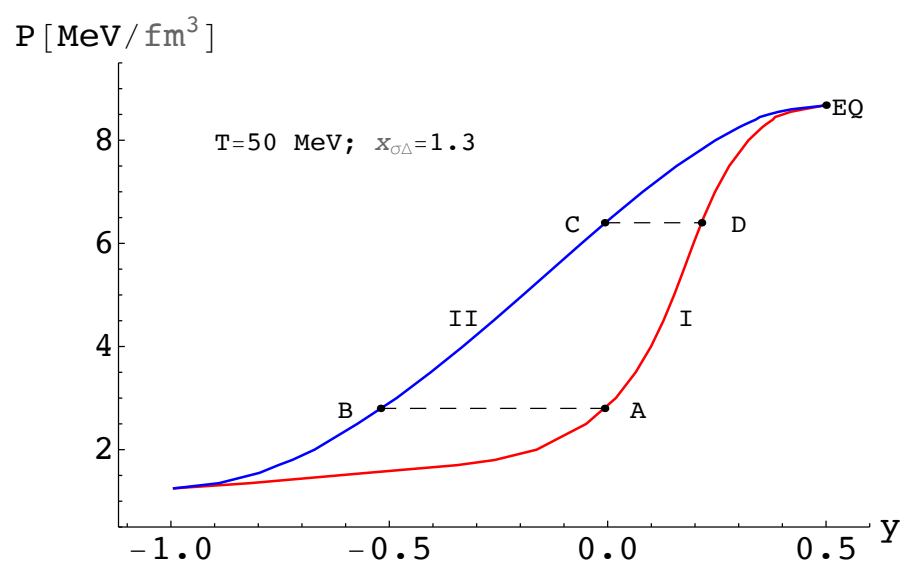

Figure 1. Binodal section at $T=50 \mathrm{MeV}$ and $x_{\sigma \Delta}=1.3$.

in $B$, at the same temperature and pressure. Let us observe that, although in $B$ the electric charge fraction is substantially negative, the relative $\Delta^{-}$abundance must be weighed on the low volume fraction occupied by the phase II near the point $B$. During the phase transition, each phase evolves towards a configuration with increasing $y$, in contrast to the liquid-gas case, where each phase evolves through a configuration with a decreasing value of $y$ (with the exception of the gas phase after the maximum asymmetry point). We will see in the next section how the presence of such features are relevant in the structure and in the evolution of the PNS.

\section{Bulk properties of the protoneutron stars}

We are going to investigate the relevance $\Delta$-isobar degrees of freedom and the stability conditions of compact star and PNS in $\beta$-stable and electric-charge neutral nuclear matter.

The neutrino mean free paths and the matter specific heat depend sensitively on the composition; under degenerate conditions even modest changes to the composition significantly alter the neutrino scattering and absorbtion mean free paths. Let us start, therefore, to investigate the particle compositions in the first leptonic rich state.

In Fig. 2, we report the particle concentrations for $s=1$ and $Y_{L}=0.4$ in presence of hyperons. The presence of $\Delta$-isobar degrees of freedom smooths the equation of state enlarging the effect of thermodynamic instabilities and altering the presence of hyperons in the PNS.

The different behavior in the stellar temperature have important consequences in the PNS evolution and in its particles concentration. Finite temperature properties of matter at high density influence the diffusion of neutrinos, being the neutrino mean free paths strongly temperature dependent [2]. In particular, neutrino opacity is very sensitive to the inner temperature (in general proportional to $T^{2}$ ) and, therefore, this would affect sensibly the cooling of the PNS.

In Fig. 3, we show the temperature as a function of the baryon density (in units of the saturation nuclear density), in absence $(n p)$ and in presence $(n p H)$ of hyperons in the SFHo model $[17,22]$. We limit our analysis in the first two phases: in the left panel, the first leptonic rich state $\left(s=1, Y_{L}=0.4\right)$ and, in the right panel, the maximum heating phase $\left(s=2, Y_{v_{e}}=0\right)$. Indeed in the cold-catalyzed phase $\left(s=0, Y_{v_{e}}=0\right)$, the temperature is very low (fews MeV), and the above statistical effects due to thermodynamical instabilities and Delta-isobar formation may be neglected. In the both previous 


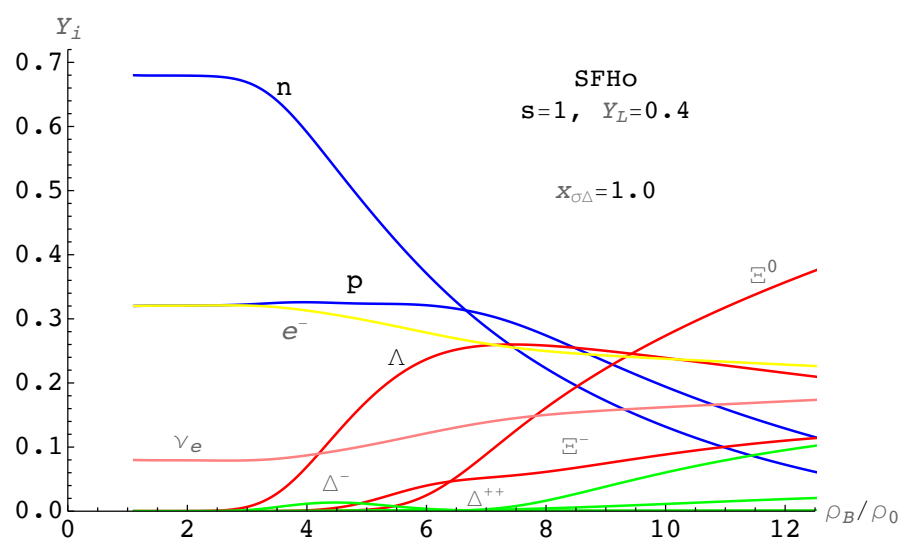

Figure 2. Particle concentrations $Y_{i}$ as a function of the baryon density at the fixed scalar coupling ratios.

cases, we observe a reduction in temperature in presence of hyperons and Delta-isobar degrees of freedom. Note also that, when hyperons are present, for $s=1$ and $Y_{L}=0.4$, the system evolves in a quasi isothermal configuration above $\rho_{B}=(2.5 \div 6) \rho_{0}$.
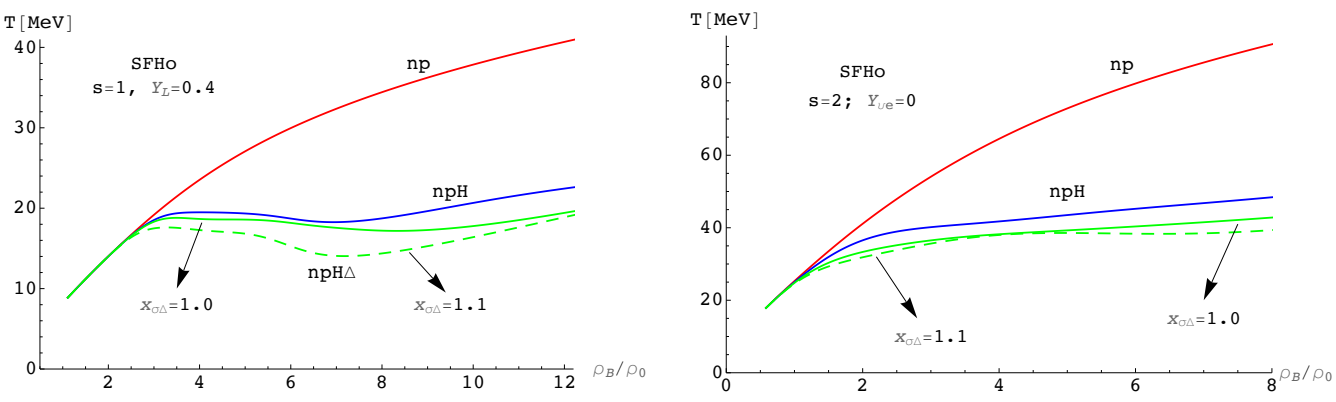

Figure 3. Temperature as a function of the baryon density (in units of the saturation nuclear density $\rho_{0}$ ) in the SFHo model and for different value of the Delta coupling constant $x_{\sigma \Delta}$. Left panel: entropy per baryon and neutrino fraction $s=1, Y_{L}=0.4$, right panel: $s=2, Y_{v_{e}}=0$. The labels $n p$ and $n p H$ stand for nucleons and nucleons plus hyperons.

In Fig. 4, we show the variation of the gravitational mass in units of solar mass $M_{\odot}$ as a function of the central baryon density $\rho_{c}$, for pure nucleonic $(n p)$ and hyperonic plus Delta-isobars $(n p H)$ stars in the first leptonic rich state (left panel, $s=1, Y_{L}=0.4$ ). For a comparison, in the figure we have considered the two models GM3 and SFHo model in presence of $\Delta$-isobars with different scalar coupling ratios $\left(x_{\sigma \Delta}=1.0\right.$ and $\left.x_{\sigma \Delta}=1.1\right)$.

Let us note the strong reduction of the gravitational mass with the introduction of hyperons and Delta-isobar degrees of freedom. This effect is remarkable stronger for a greater value of the $x_{\sigma \Delta}$ coupling due also to the presence of thermodynamical instabilities conditions.

In the presence of hyperons, when the stellar core contains non-leptonic negative charges, the maximum masses of neutrino-trapped stars result to be significantly larger than for low temperatures 


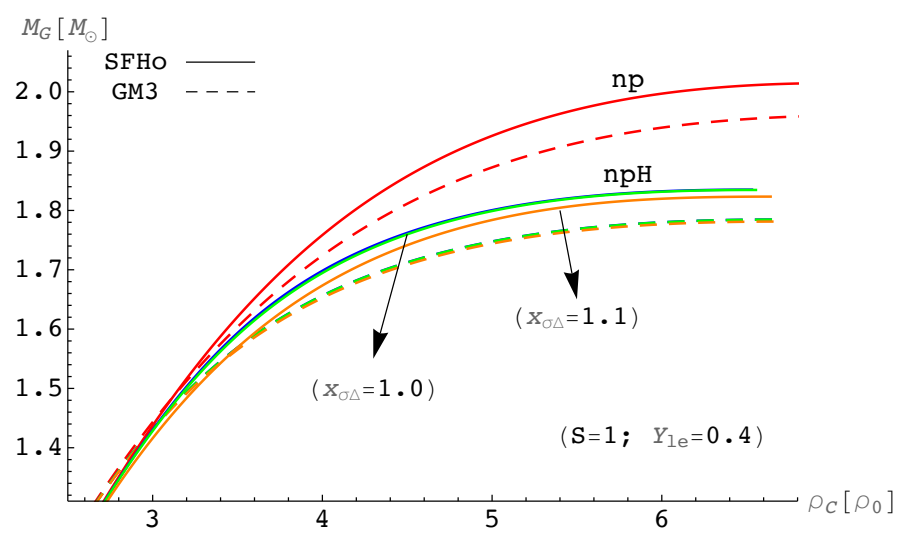

Figure 4. Gravitational mass $M_{G}$ in units of solar mass $M_{\odot}$ as a function of the central baryon density $\rho_{c}$ (in units of the nuclear saturation density $\rho_{0}$ ) for nucleons (np) and hyperons stars (npH) stars in the case $s=1$ and $Y_{L}=0.4$.

and for lepton poor matter [2]. Hence, there exists a window of initial masses for which the star becomes unstable to gravitational collapse during deleptonization and a black hole can take place.

\section{References}

[1] M. Prakash et al., Lect. Notes Phys. 578364 (2001).

[2] J.A. Pons, A.W. Steiner, M. Prakash, J.M. Lattimer, Phys. Rev. Lett. 86, 5223 (2001).

[3] A. Lavagno, D. Pigato, Eur. Phys. J. A 4752 (2011).

[4] N.K. Glendenning, Phys. Rev. D 461274 (1992).

[5] A. Lavagno, Phys. Rev. C 81044909 (2010).

[6] A. Lavagno, D. Pigato, J. Phys. G: Nucl. Part. Phys. 39125106 (2012).

[7] M. Hofmann et al., Phys. Rev. C 512095 (1995).

[8] Z. Li, G. Mao, Y. Zhuo, W. Greiner, Phys. Rev. C 561570 (1997).

[9] G. Mao, L. Neise, H. Stöcker, W. Greiner, Phys. Rev. C 59, 1674 (1999).

[10] P. Fachini, J. Phys. G: Nucl. Part. Phys. 35, 044032 (2008).

[11] A. Lavagno, D. Pigato, Phys. Rev. C 86024917 (2012).

[12] A. Lavagno, Eur. Phys. J. A 49102 (2013).

[13] A. Lavagno, D. Pigato, P. Quarati, J. Phys. G: Nucl. Part. Phys. 37, 115102 (2010).

[14] A. Drago, A. Lavagno, G. Pagliara, D. Pigato, Phys. Rev. C 90065809 (2014).

[15] N.K. Glendenning, S.A. Moszkowski, Phys. Rev. Lett. 67, 2414 (1991).

[16] A. Drago, A. Lavagno, P. Quarati, Physica A 344472 (2004).

[17] A.W. Steiner, M. Hempel, T. Fischer, Astrophys. J. 77417 (2013).

[18] A. Drago, A. Lavagno, G. Pagliara, Phys. Rev. D 89043014 (2014).

[19] H. Müller, B.D. Serot, Phys. Rev. C 522072 (1995).

[20] A. Lavagno, P. Narayana Swamy, Found. Phys. 40814 (2010).

[21] A. Lavagno, P. Narayana Swamy, Physica A 389993 (2010).

[22] A. Drago et al., Phys. Rev. D 93103001 (2016). 